\title{
Scheme to Measure Quantum Stokes Parameters and their Fluctuations and Correlations
}

\author{
G. S. Agarwal* \\ Physical Research Laboratory, Navrangpura, Ahmedabad 380009, India \\ S. Chaturvedi ${ }^{\dagger}$ \\ School of Physics, University of Hyderabad, Hyderabad 500046, India
}

(October 24, 2018)

\begin{abstract}
We propose a scheme to measure quantum Stokes parameters, their fluctuations and correlations. The proposal involves measurements of intensities and intensity-intensity correlations for suitably defined modes, which can be produced by a combination of half wave and quarter wave plates.
\end{abstract}

*email: gsa@prl.ernet.in

†email: scsp@uohyd.ernet.in 
Polarisation of light is a well studied subject. Text book [1] descriptions of light polarisation are based on the Stokes parameters. Consider, for example, a light beam propagating in $z$ direction and decomposed into two components polarised along the orthogonal directions $\hat{\epsilon}_{i}, i=1,2$ :

$$
\vec{E}(z, t) \equiv\left(\hat{\epsilon}_{1} \alpha_{1}+\hat{\epsilon}_{2} \alpha_{2}\right) e^{i k z-i w t}+\quad \text { c.c. } .
$$

The four Stokes parameters characterising such a beam are defined by [1]

$$
\begin{aligned}
& S_{0}=\left|\alpha_{1}\right|^{2}+\left|\alpha_{2}\right|^{2} \quad ; \quad S_{1}=\left|\alpha_{1}\right|^{2}-\left|\alpha_{2}\right|^{2} ; \\
& S_{2}=\left(\alpha_{1}^{*} \alpha_{2}+\alpha_{1} \alpha_{2}^{*}\right) \quad ; \quad S_{3}=-i\left(\alpha_{1}^{*} \alpha_{2}-\alpha_{1} \alpha_{2}^{*}\right),
\end{aligned}
$$

which, as is well known, lead to the representation of light polarisation by a point on the Poincaré sphere. All states of linear polarisation lie in the $x y$ plane, whereas north and south poles represent states of circular polarisation. All other points on Poincaré sphere represent states of elliptic polarisation. Methods to measure all four Stokes parameters are also well known.

The Stokes parameters have been generalised in several ways [2]. For example Wolf [3] has shown how the polarisation characteristics of partially coherent light can be calculated. In such a case one has to average quantities defined by (2), over an ensemble distribution of $\alpha_{1}$ and $\alpha_{2}$. More recently, there has been considerable interest in generalising these ideas to discuss the polarisation characteristics of a non classical field possessing very strong quantum fluctuations [4.5]. In such a case the field $\vec{E}$ in (1) becomes an operator with $\alpha_{1}$ and $\alpha_{2}$ replaced by the annihilation operators $a_{1}$ and $a_{2}$. We can then define quantum Stokes operators [7], in analogy to (2), as :

$$
\begin{aligned}
& \mathcal{S}_{0}=\left(a_{1}^{\dagger} a_{1}+a_{2}^{\dagger} a_{2}\right), \\
& \mathcal{S}_{1}=\left(a_{1}^{\dagger} a_{1}-a_{2}^{\dagger} a_{2}\right), \\
& \mathcal{S}_{2}=\left(a_{1}^{\dagger} a_{2}+a_{2}^{\dagger} a_{1}\right), \\
& \mathcal{S}_{3}=-i\left(a_{1}^{\dagger} a_{2}-a_{2}^{\dagger} a_{1}\right) ;\left[a_{i}, a_{j}^{\dagger}\right]=\delta_{i j} .
\end{aligned}
$$

The expectation values $\left\langle\mathcal{S}_{i}\right\rangle, i=0,1,2,3$ can be identified with the Stokes parameters $S_{i}, i=0,1,2,3$.

As is well known, the above operators furnish the Schwinger representation [8] of the $S U(2)$ algebra

$$
\begin{aligned}
{\left[\mathcal{S}_{i}, \mathcal{S}_{j}\right] } & =2 i \epsilon_{i j k} \mathcal{S}_{k}, i, j, k=1,2,3 \\
{\left[\mathcal{S}_{0}, \mathcal{S}_{i}\right] } & =0, i=1,2,3 \\
\mathcal{S}_{0}\left(\mathcal{S}_{0}+2\right) & =\mathcal{S}_{1}^{2}+\mathcal{S}_{2}^{2}+\mathcal{S}_{3}^{2} .
\end{aligned}
$$

The connection to the $S U(2)$ algebra is very useful and many of the concepts developed in connection with spin squeezing [9] have been taken over to discuss polarisation squeezing [4,5,10,11]. The Stokes parameters $S_{i}, i=0,1,2,3$ involve four averages $\left\langle a_{1}^{\dagger} a_{1}\right\rangle,\left\langle a_{2}^{\dagger} a_{2}\right\rangle,\left\langle a_{1}^{\dagger} a_{2}\right\rangle,\left\langle a_{2}^{\dagger} a_{1}\right\rangle$. Further, the variances

$$
V_{i j}=\frac{1}{2}\left(\left\langle\left\{\mathcal{S}_{i}, \mathcal{S}_{j}\right\}\right\rangle-\left\{\left\langle\mathcal{S}_{i}\right\rangle,\left\langle\mathcal{S}_{j}\right\rangle\right\}\right) ; i, j=0, \cdots, 3, i \leq j,
$$


can be expressed in terms of the nine normally ordered field correlations $\left\langle a_{1}^{\dagger} a_{1}^{\dagger} a_{1} a_{1}\right\rangle,\left\langle a_{2}^{\dagger} a_{2}^{\dagger} a_{2} a_{2}\right\rangle,\left\langle a_{1}^{\dagger} a_{1}^{\dagger} a_{2} a_{2}\right\rangle,\left\langle a_{2}^{\dagger} a_{2}^{\dagger} a_{1} a_{1}\right\rangle,\left\langle a_{1}^{\dagger} a_{2}^{\dagger} a_{1} a_{2}\right\rangle,\left\langle a_{1}^{\dagger} a_{1}^{\dagger} a_{1} a_{2}\right\rangle,\left\langle a_{2}^{\dagger} a_{2}^{\dagger} a_{1} a_{2}\right\rangle,\left\langle a_{1}^{\dagger} a_{2}^{\dagger} a_{1} a_{1}\right\rangle$, $\left\langle a_{1}^{\dagger} a_{2}^{\dagger} a_{2} a_{2}\right\rangle$. Given the circumstance that, in an experiment, one typically measures average intensities and intensity variances, the question one is confronted with is whether it is possible to deduce the relevant field correlations by measuring average intensities and intensity variances of suitably defined modes. The answer to this question is provided by a theorem due to Mukunda and Jordan [12] in a more general context. This would be used in the following to show how all $V_{i j}$ can be essentially obtained from intensities and intensity fluctuations. We note that very recently Korolkova et al [⿴囗⿴囗十 have proposed a method to measure the diagonal quantities $V_{i i}$. An implementation of this proposal has been achieved by Bowen et al [13].

Before we proceed further, we note that for an important special class of the states of the radiation field, such as the squeezed coherent state $\left|\alpha_{1}, \alpha_{2}, \zeta\right\rangle$, the correlations can be derived in closed analytical form. In this case it is well known that the Wigner function as well as the $Q$ - function are Gaussian [14]. Thus the fourth order expectation values can be expressed in terms of second order expectation values. If we define displaced modes $d_{i}=$ $a_{i}-\alpha_{i}$, then we have for example

$$
\begin{aligned}
\left\langle d_{1} d_{2} d_{1}^{\dagger} d_{2}^{\dagger}\right\rangle & \equiv\left\langle d_{1} d_{2}\right\rangle\left\langle d_{1}^{\dagger} d_{2}^{\dagger}\right\rangle+\left\langle d_{1} d_{1}^{\dagger}\right\rangle\left\langle d_{2} d_{2}^{\dagger}\right\rangle+\left\langle d_{1} d_{2}^{\dagger}\right\rangle\left\langle d_{2} d_{1}^{\dagger}\right\rangle \\
& =\left\langle d_{1} d_{2}\right\rangle\left\langle d_{1}^{\dagger} d_{2}^{\dagger}\right\rangle+\left\langle d_{1} d_{1}^{\dagger}\right\rangle\left\langle d_{2} d_{2}^{\dagger}\right\rangle
\end{aligned}
$$

since for the squeezed state $\left\langle d_{1} d_{2}^{\dagger}\right\rangle=0$. It should be borne in mind that the special case of coherent states is obtained by setting $\zeta=0$. Clearly if one knows, a priori, that the field is in the squeezed state then all the fluctuations and correlations among Stokes parameters can be related to second order expectation values. For arbitrary non classical states characterised by non-Gaussian Wigner function, the situation would be much more complex. Another simple case is obtained if the fluctuations of the field around the mean value are small. In such a case $S_{k}-\left\langle S_{k}\right\rangle$ can be approximated by terms linear in $d_{i}$ and $d_{i}^{\dagger}$. Thus correlations $V_{i j}$ are expressible in terms of the expectation values of quadratic forms. The latter, in many cases reduces to variances in quadratures [1, 13].

As noted earlier, The measurement scheme proposed here is based on a work of Mukunda and Jordan [12] which, In the present context, involves use of unitary transformations defined by

$$
\left(\begin{array}{l}
b_{1} \\
b_{2}
\end{array}\right)=u\left(\begin{array}{l}
a_{1} \\
a_{2}
\end{array}\right) ; u=\left(\begin{array}{cc}
\cos \theta & e^{i \phi} \sin \theta \\
-e^{i \phi} \sin \theta & \cos \theta
\end{array}\right)
$$

and measurements of the intensities $\left\langle b_{i}^{\dagger} b_{i}\right\rangle(i=1,2)$ and intensity correlations like $\left\langle b_{1}^{\dagger} b_{1}^{\dagger} b_{1} b_{2}\right\rangle,\left\langle b_{1}^{\dagger} b_{2}^{\dagger} b_{1} b_{2}\right\rangle$ for different settings of $\theta, \phi$. Thus, for the mean intensity, we have

$$
\begin{aligned}
\left\langle b_{1}^{\dagger}(u) b_{1}(u)\right\rangle & =\cos ^{2} \theta\left\langle a_{1}^{\dagger} a_{1}\right\rangle+\sin ^{2} \theta\left\langle a_{2}^{\dagger} a_{2}\right\rangle \\
& +e^{i \phi} \sin \theta \cos \theta\left\langle a_{1}^{\dagger} a_{2}\right\rangle+e^{-i \phi} \sin \theta \cos \theta\left\langle a_{2}^{\dagger} a_{1}\right\rangle .
\end{aligned}
$$

We can, therefore, determine $\left\langle a_{1}^{\dagger} a_{1}\right\rangle,\left\langle a_{2}^{\dagger} a_{2}\right\rangle,\left\langle a_{1}^{\dagger} a_{2}\right\rangle,\left\langle a_{2}^{\dagger} a_{1}\right\rangle$ from the knowledge of $\left\langle b_{1}^{\dagger}(u) b_{1}(u)\right\rangle$, for instance, for the four settings corresponding to $(\theta, \phi)$ equal to $(0,0),(\pi / 2,0),(\pi / 4,0),(\pi / 4, \pi / 2)$. This is, in fact, identical to the procedure adopted 
for measurement of classical stokes parameters [1].

Next we turn to the nine field correlations which arise in the expressions for the variances of the Stokes operators. On selecting the unitary operator in (8) as

$$
u=\left(\begin{array}{cc}
\cos \theta & \sin \theta \\
-\sin \theta & \cos \theta
\end{array}\right)
$$

we find the relation

$$
\begin{aligned}
\left\langle b_{1}^{\dagger}(u) b_{1}^{\dagger}(u) b_{1}(u) b_{1}(u)\right\rangle & =\left\langle a_{1}^{\dagger} a_{1}^{\dagger} a_{1} a_{1}\right\rangle \cos ^{4} \theta+\left\langle a_{2}^{\dagger} a_{2}^{\dagger} a_{2} a_{2}\right\rangle \sin ^{4} \theta \\
& +\left(4\left\langle a_{1}^{\dagger} a_{2}^{\dagger} a_{1} a_{2}\right\rangle+\left\langle a_{1}^{\dagger} a_{1}^{\dagger} a_{2} a_{2}\right\rangle+\left\langle a_{2}^{\dagger} a_{2}^{\dagger} a_{1} a_{1}\right\rangle\right) \cos ^{2} \theta \sin ^{2} \theta \\
& +2\left(\left\langle a_{1}^{\dagger} a_{1}^{\dagger} a_{1} a_{2}\right\rangle+\left\langle a_{1}^{\dagger} a_{2}^{\dagger} a_{1} a_{1}\right\rangle\right) \cos ^{3} \theta \sin \theta \\
& +2\left(\left\langle a_{1}^{\dagger} a_{2}^{\dagger} a_{2} a_{2}\right\rangle+\left\langle a_{2}^{\dagger} a_{2}^{\dagger} a_{1} a_{2}\right\rangle\right) \cos \theta \sin ^{3} \theta,
\end{aligned}
$$

which for five different values of $\theta$ allows us to determine the terms with different dependence on $\theta$ in terms of the measured values $\left\langle b_{1}^{\dagger}(u) b_{1}^{\dagger}(u) b_{1}(u) b_{1}(u)\right\rangle$ for the chosen $\theta$ settings. Next taking the unitary operator as

$$
u=\left(\begin{array}{cc}
\cos \theta & i \sin \theta \\
+i \sin \theta & \cos \theta
\end{array}\right)
$$

we get the relation

$$
\begin{aligned}
\left\langle b_{1}^{\dagger}(u) b_{1}^{\dagger}(u) b_{1}(u) b_{1}(u)\right\rangle & =\left\langle a_{1}^{\dagger} a_{1}^{\dagger} a_{1} a_{1}\right\rangle \cos ^{4} \theta+\left\langle a_{2}^{\dagger} a_{2}^{\dagger} a_{2} a_{2}\right\rangle \sin ^{4} \theta \\
& +\left(4\left\langle a_{1}^{\dagger} a_{2}^{\dagger} a_{1} a_{2}\right\rangle-\left\langle a_{1}^{\dagger} a_{1}^{\dagger} a_{2} a_{2}\right\rangle-\left\langle a_{2}^{\dagger} a_{2}^{\dagger} a_{1} a_{1}\right\rangle\right) \cos ^{2} \theta \sin ^{2} \theta \\
& +2 i\left(\left\langle a_{1}^{\dagger} a_{1}^{\dagger} a_{1} a_{2}\right\rangle-\left\langle a_{1}^{\dagger} a_{2}^{\dagger} a_{1} a_{1}\right\rangle\right) \cos ^{3} \theta \sin \theta \\
& +2 i\left(\left\langle a_{1}^{\dagger} a_{2}^{\dagger} a_{2} a_{2}\right\rangle-\left\langle a_{2}^{\dagger} a_{2}^{\dagger} a_{1} a_{2}\right\rangle\right) \cos \theta \sin ^{3} \theta .
\end{aligned}
$$

Here, three suitably chosen values of $\theta$ are sufficient to determine the terms with different dependence on $\theta$ since the first two terms were determined previously. Finally, choosing

$$
u=\frac{1}{\sqrt{2}}\left(\begin{array}{cc}
1 & e^{i \pi / 4} \\
-e^{-i \pi / 4} & 1
\end{array}\right)
$$

we obtain

$$
\begin{aligned}
\left\langle b_{1}^{\dagger}(u) b_{2}^{\dagger}(u) b_{1}(u) b_{2}(u)\right\rangle & =\frac{1}{4}\left\langle a_{1}^{\dagger} a_{1}^{\dagger} a_{1} a_{1}\right\rangle+\frac{1}{4}\left\langle a_{2}^{\dagger} a_{2}^{\dagger} a_{2} a_{2}\right\rangle \\
& -\frac{i}{4}\left(\left\langle a_{1}^{\dagger} a_{1}^{\dagger} a_{2} a_{2}\right\rangle-\left\langle a_{2}^{\dagger} a_{2}^{\dagger} a_{1} a_{1}\right\rangle\right) .
\end{aligned}
$$

Thus the nine choices of $u$ above permit us to determine the nine field correlations listed after Eq.(5) and hence all the variances in the Stokes operators. Besides the variances, the same nine correlations determine the correlations among Stokes operators. For example the last term in Eq.(15) represents the normally ordered correlation $\left\langle{ }_{0}^{\circ} S_{2} S_{3}^{\circ}{ }_{\circ}^{\circ}\right\rangle$. Further note that (11) [(13)] for $\theta=\pi / 4$ and $\theta=3 \pi / 4$ when added and subtracted would yield

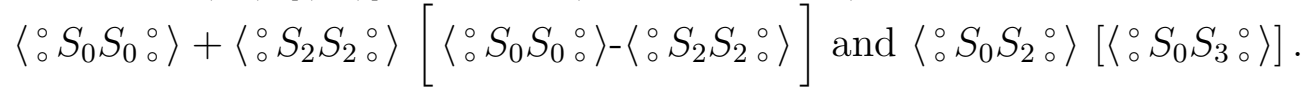


The unitary transformations $u$ involved in the measurements above are all of the form

$$
u(\theta, \phi)=\left(\begin{array}{cc}
\cos \theta & e^{i \phi} \sin \theta \\
-e^{-i \phi} \sin \theta & \cos \theta
\end{array}\right),
$$

with $\phi$ taking values $0, \pi / 4, \pi / 2$. These transformations can most conveniently be realized using the universal $S U(2)$ gadget [15, 16] which consists of two quarter wave plates and one half wave plate mounted coaxially. Taking the axis of the gadget to be along the $z$ axis and the slow axes of the quarter and half wave plates in the $x$ direction, the settings for the $S U(2)$ gadget in the $\mathrm{Q}-\mathrm{Q}-\mathrm{H}$ configuration which realize the required transformations are

$$
\begin{aligned}
u(\theta, 0) & =Q_{\pi / 4} Q_{\pi / 4} H_{-\pi / 4+\theta / 2}, \\
u(\theta, \pi / 2) & =Q_{\pi / 2} Q_{(\theta+\pi / 2)} H_{\theta / 2}, \\
u(\pi / 4, \pi / 4) & =Q_{\pi / 4+\left(\tan ^{-1} \sqrt{2}\right) / 2} Q_{5 \pi / 12+\left(\tan ^{-1} \sqrt{2}\right) / 2} H_{\pi / 12},
\end{aligned}
$$

where the the subscripts to $Q$ and $H$ give the angles by which the quarter and the half wave plates have to be rotated around the $z$ axis. The matrices for the quarter wave and half wave plates are given by

$$
\begin{aligned}
H_{\phi} & =i\left(\begin{array}{cc}
\cos 2 \phi & \sin 2 \phi \\
\sin 2 \phi & -\cos 2 \phi
\end{array}\right), \\
Q_{\phi} & =\frac{i}{\sqrt{2}}\left(\begin{array}{cc}
\cos 2 \phi-i & \sin 2 \phi \\
\sin 2 \phi & -\cos 2 \phi-i
\end{array}\right) .
\end{aligned}
$$

After 'rotating' the $a_{1}$ and $a_{2}$ into $b_{1}(u)$ and $b_{2}(u)$ modes with the help of the $S U(2)$ gadget one can separate the $b_{1}(u), b_{2}(u)$ modes with the help of a polarising beam splitter and carry out the required intensity and intensity-intensity correlation measurements to obtain complete information about the Stokes parameters and fluctuations thereof.

To conclude, we have shown how a combination of quarter wave and half wave plates can be used to measure not only the Stokes parameters but also all the quantum correlations and fluctuations in the Stokes parameters. 


\section{REFERENCES}

[1] M. Born and E. Wolf, Principles of Optics, Seventh Edition, (Cambridge University Press 1999), Sec.1.4.

[2] L. Mandel and E. Wolf, "Optical Coherence and Quantum Optics" (Cambridge University Press, 1995), Chap.6.

[3] E. Wolf, Nuovo Cimento 13, 1165 (1959).

[4] N. V. Korolkova, G. Leuchs, R. Loudon, T. C. Ralph and C. Silberhorn, quant$\mathrm{ph} / 0108098$.

[5] N. V. Korolkova and A. S. Chirkin, J. Mod. Opt. 43, 869 (1996).

[6] . A. F. Abouraddy, A. V. Sergienko, B. E. A. Saleh and M. C. Teich, Opt. Commun. 201, 93 (2002); D. F. V. James, P. G. Kwiat, W. J. Munro and A. G. White, Phys. Rev. A 64, 052312 (2001).

[7] J. M. Jauch and F. Rohrlich, The Theory of Photons and Electrons (Springer-Verlag, Berlin 1980) Sec.2.8. B.A. Robson, The Theory of Polarization Phenomena (Clarendon Press, Oxford 1974).

[8] J. Schwinger, Proc. Nat. Acad. Sci. U.S.A 46, 570 (1960).

[9] G. S.Agarwal and R. R. Puri, Phys. Rev. A 41, 3782 (1990); ibid 49, 4968 (1994); A. Kuzmich, L. Mandel and N. P. Bigelow, Phys. Rev. Lett. 85, 1594 (2000), A. Sorensen and K. Molmer quant-ph/0011035.

[10] P. Grangier, R. E. Slusher, B. Yurke and A. LaPorta, Phys. Rev. Lett. 59, 2153 (1987).

[11] A .P. Alodjants, A. M. Arakelian and A. S. Chirkin, Appl. Phys. B 66, 53 (1998); G. S. Agarwal and R. R. Puri, Phys. Rev. A 40, 5179 (1989).

[12] N. Mukunda and T. F. Jordan, J. Math. Phys. 7, 849 (1966).

[13] W. P. Bowen, R. Schnabel, Hans A. Bachor and P. K. Lam, Phys. Rev. Lett. 88, 093601 (2002).

[14] D. F. Walls and G. J. Milburn, Quantum Optics (Springer-Verlag, Berlin, 1994); Chap.V.

[15] R. Simon and N. Mukunda, Phys. Lett. A 138, 474 (1990).

[16] R. Simon and N. Mukunda, Phys. Lett. A 143, 165 (1990). 